\title{
Prognosis after percutaneous coronary intervention in patients with psoriasis: a cohort study using Danish nationwide registries
}

\author{
Ole Ahlehoff ${ }^{1,2 *}$, Jesper Lindhardsen', Gunnar H Gislason ${ }^{1}$, Jonas B Olesen ${ }^{1}$, Mette Charlot ${ }^{1}$, Lone Skov ${ }^{3}$, \\ Christian Torp-Pedersen ${ }^{1}$ and Peter $\mathrm{R}$ Hansen ${ }^{1}$
}

\begin{abstract}
Background: Psoriasis is an inflammatory disease associated with increased risk of coronary artery disease. However, the potential impact of psoriasis on the prognosis following percutaneous coronary revascularization (PCl) is unknown.

Methods: The study comprised the entire Danish population undergoing first-time PCl in the period 2002-09. Cox regression models, controlling for age, gender, socioeconomic status, pharmacological treatment, and comorbidity were used to assess the risk of 1) all-cause mortality and 2) a composite endpoint of death, myocardial infarction, and stroke.

Results: A total of 53,141 patients with first-time PCl in the study period were identified. Of these, 1074 had mild psoriasis and 315 had severe psoriasis. Patients with severe psoriasis, but not those with mild disease had increased risk of both endpoints compared to patients without psoriasis. The incidence rates for all-cause mortality were 30.5 (Cl 29.7-31.3), 29.9 (Cl 24.7-36.1), and 47.2 (Cl 35.0-63.6) per 1000 patient years for patients without psoriasis, with mild psoriasis, and with severe psoriasis, respectively. Hazard ratios were $1.10(\mathrm{Cl} 0.91-1.33)$ and $1.67(\mathrm{Cl} 1.24-2.26)$ for mild and severe psoriasis, respectively. Patients with severe psoriasis were less likely to receive secondary prevention pharmacotherapy with betablockers, statins and platelet inhibitors.

Conclusion: This first study of the prognosis following PCI in patients with psoriasis demonstrated an increased risk of all-cause mortality and of a composite of death, myocardial infarction and stroke, respectively, in patients with severe psoriasis compared to patients without psoriasis. Further studies of this novel association are needed.
\end{abstract}

Keywords: Coronary revascularization, Cardiovascular disease, Inflammation, Psoriasis, Prognosis

\section{Background}

Psoriasis is a chronic inflammatory disease affecting the skin of $1-3 \%$ of the population [1]. Other manifestations of psoriasis include scalp psoriasis, nail disease, and sero-negative arthritis known as psoriatic arthritis [1]. Atherosclerosis is also regarded as an inflammatory disease and the underlying mechanisms are very similar to those seen in psoriasis [2,3]. Furthermore, psoriasis is

\footnotetext{
* Correspondence: olahha01@geh.regionh.dk

'Department of Cardiology, Copenhagen University Hospital, Gentofte, Hellerup DK-2900, Denmark

${ }^{2}$ Department of Cardiology, Copenhagen University Hospital, Roskilde,

Roskilde DK-4000, Denmark

Full list of author information is available at the end of the article
}

associated with conventional cardiovascular risk factors, including diabetes mellitus, hypertension, and dyslipidemia $[4,5]$. Like other inflammatory disorders including rheumatoid arthritis and systemic lupus erythromatosus, psoriasis confers independent risk of atherothrombotic disease, invasive coronary revascularization, and cardiovascular mortality [6-11]. In the Danish population, patients with psoriasis have been demonstrated to have an increased (1.4 to 2.3 fold) risk of coronary revascularization, including percutaneus coronary intervention (PCI) [9]. Recent results have indicated that patients with psoriasis face a poorer prognosis following myocardial infarction compared to patients without psoriasis [12]. Although PCI is widely used in the treatment of acute

\section{Biomed Central}


and chronic coronary artery disease there are no data available on the potential impact of psoriasis on post-PCI prognosis.

We therefore used the Danish nationwide registries to examine the psoriasis-related risks of death, myocardial infarction, stroke, and repeat revascularization in patients with and without psoriasis after first-time PCI in the time-period 2002-2009.

\section{Results}

Baseline characteristics of the study population are presented in Table 1. A total of 53,141 patients, including 1074 with mild psoriasis and 315 with severe psoriasis, with first-time PCI in the study period were identified. Patients with severe psoriasis were slightly younger and more often women compared to patients without psoriasis. In approximately $55-60 \%$ the index PCIs were performed in patients with a previous myocardial infarction, including $26-29 \%$ primary PCI procedures, with no significant differences between patients with severe psoriasis and patients without psoriasis (Tables 1 and 2). In the majority (88.3\%) of baseline previous myocardial infarctions the event date was less than 30 days from the index PCI. At baseline, patients with psoriasis received more non steroid anti-inflammatory drugs (NSAIDs) and more antihypertensive, cholesterol-lowering, glucose-lowering, and antidepressive medication, respectively, than patients without psoriasis.

Patients who survived to enter the sensitivity analyses with follow-up start at day 30 after the index PCI had a similar gender distribution and the prevalence of psoriasis in this analysis was similar (2.7\%). There were also no differences in the proportion of (index) primary PCI procedures between the groups in this population. Patients with severe psoriasis generally received significantly less secondary prevention medication including beta-blockers, statins, and platelet inhibitors. Furthermore, as in the

Table 1 Baseline characteristics of the study population

\begin{tabular}{|c|c|c|c|c|}
\hline & No psoriasis & Mild psoriasis & Severe psoriasis & p-value \\
\hline & $n=51,752$ & $\mathrm{n}=1074$ & $n=315$ & \\
\hline Male (\%) & $36,989(71.5)$ & 767 (71.4) & $193(61.3)$ & 0.003 \\
\hline Female (\%) & $14,763(28.5)$ & 307 (28.6) & $122(38.7)$ & \\
\hline Age, years (SD) & $65.1(11.6)$ & $65.0(10.7)$ & $63.5(10.9)$ & 0.06 \\
\hline \multicolumn{5}{|l|}{ Treatment (\%) } \\
\hline Beta-blocker & $20,851(40.3)$ & $434(40.4)$ & $132(41.9)$ & 0.60 \\
\hline ACE inhibitor/ARB & $16,868(32.6)$ & $381(35.5)$ & $121(38.4)$ & 0.003 \\
\hline Statin & $21,462(41.5)$ & $506(47.1)$ & $136(43.2)$ & 0.003 \\
\hline Platelet inhibitor & $21,209(41.0)$ & $490(45.6)$ & $133(42.2)$ & 0.02 \\
\hline Loop diuretic & $6663(12.9)$ & $139(12.9)$ & $48(15.2)$ & 0.33 \\
\hline Spironolactone & $1733(3.4)$ & $38(3.5)$ & $11(3.5)$ & 0.74 \\
\hline Vitamin $\mathrm{K}$ antagonist & $2505(4.8)$ & $48(4.5)$ & $11(3.5)$ & 0.23 \\
\hline Glucose lowering medication & $5734(11.1)$ & $123(11.5)$ & $52(16.5)$ & 0.01 \\
\hline NSAID & $3465(6.7)$ & $101(9.4)$ & $36(11.4)$ & $<0.001$ \\
\hline Antidepressive medication & $4569(8.8)$ & $125(11.6)$ & $42(13.3)$ & $<0.001$ \\
\hline \multicolumn{5}{|l|}{ Comorbidity (\%) } \\
\hline Myocardial infarction & $31,078(60.1)$ & $596(55.5)$ & $182(60.0)$ & 0.008 \\
\hline Cardiac dysrhythmia & $4759(9.2)$ & $115(10.7)$ & $28(3.2)$ & 0.33 \\
\hline Diabetes with complications & $2117(4.1)$ & $38(3.5)$ & $22(7.0)$ & 0.20 \\
\hline Cerebrovascular disease & $1373(2.7)$ & $33(3.1)$ & $10(3.2)$ & 0.32 \\
\hline Pulmonary edema & $187(0.4)$ & $5(0.5)$ & $2(0.6)$ & 0.33 \\
\hline Shock & $593(1.2)$ & $6(0.6)$ & $8(2.5)$ & 0.62 \\
\hline Cancer & $424(0.8)$ & $9(0.8)$ & $3(1.0)$ & 0.62 \\
\hline Acute renal failure & $331(0.6)$ & $2(0.2)$ & $3(1.0)$ & 0.46 \\
\hline Chronic renal failure & $592(1.1)$ & $8(0.2)$ & $4(1.1)$ & 0.51 \\
\hline Chronic renal failure & $1904(3.7)$ & $50(4.7)$ & $15(4.8)$ & 0.06 \\
\hline pulmonary disease & & & & \\
\hline
\end{tabular}

ACE: angiotensin-converting enzyme inhibitor; ARB: angiotensin 2 receptor blocker; NSAID: Non-steroid anti-inflammatory drug. 
Table 2 Post $\mathrm{PCl}$ characteristics at 30, 180, and 360 days from index $\mathrm{PCI}$

\begin{tabular}{|c|c|c|c|c|}
\hline \multirow[t]{2}{*}{30 days after $\mathrm{PCl}$} & No psoriasis & Mild psoriasis & Severe psoriasis & p-value \\
\hline & $n=49,696$ & $n=1027$ & $n=309$ & \\
\hline Male (\%) & $35,632(72.7)$ & $737(72.8)$ & $192(62.1)$ & 0.007 \\
\hline Female (\%) & $14,064(28.3)$ & $290(28.2)$ & $117(37.9)$ & \\
\hline Primary PCI (\%) & $14,451(29.1)$ & $268(26.1)$ & $90(29.1)$ & 0.17 \\
\hline \multicolumn{5}{|l|}{ Treatment (\%) } \\
\hline Beta-blocker & $38,916(78.3)$ & $772(75.2)$ & $230(74.4)$ & 0.005 \\
\hline ACE inhibitor/ARB & $25,032(50.4)$ & $509(49.6)$ & $167(54.1)$ & 0.54 \\
\hline Statin & $42,844(86.2)$ & $896(86.3)$ & $245(79.3)$ & 0.049 \\
\hline Platelet inhibitor & $47,949(96.5)$ & $991(96.5)$ & $285(92.2)$ & 0.003 \\
\hline Dual anti-platelet inhibition & $45,901(92.4)$ & $946(92.1)$ & $273(88.4)$ & 0.029 \\
\hline Loop diuretic & $9812(19.7)$ & $201(19.6)$ & $71(23.0)$ & 0.34 \\
\hline Spironolactone & $2852(5.7)$ & $53(5.2)$ & $23(7.4)$ & 0.67 \\
\hline Vitamin $\mathrm{K}$ antagonist & $2904(5.8)$ & $58(5.7)$ & $18(5.8)$ & 0.85 \\
\hline Glucose-lowering medication & $5586(11.2)$ & $112(10.9)$ & $52(16.8)$ & 0.039 \\
\hline NSAID & $1349(2.7)$ & $40(3.9)$ & $34(11.0)$ & 0.002 \\
\hline \multirow[t]{2}{*}{180 days after $\mathrm{PCl}$} & No psoriasis & Mild psoriasis & Severe psoriasis & p-value \\
\hline & $n=46,126$ & $\mathrm{n}=955$ & $n=279$ & \\
\hline Beta-blocker & $36,431(78.9)$ & $727(76.1)$ & $212(76.0)$ & 0.05 \\
\hline ACE inhibitor/ARB & 23,324 (50.6) & $484(50.7)$ & $153(54.8)$ & 0.36 \\
\hline Statin & $40,140(87.0)$ & $842(88.2)$ & $226(81.0)$ & 0.007 \\
\hline Platelet inhibitor & $44,696(96.9)$ & $927(97.1)$ & $260(93.2)$ & 0.002 \\
\hline Dual anti-platelet inhibition & $42,822(92.8)$ & $889(93.1)$ & $249(89.3)$ & 0.07 \\
\hline Loop diuretic & 8968 (19.4) & $188(19.7)$ & $62(22.2)$ & 0.50 \\
\hline \multirow[t]{2}{*}{360 days after $\mathrm{PCl}$} & No psoriasis & Mild psoriasis & Severe psoriasis & p-value \\
\hline & $n=42,188$ & $n=860$ & $\mathrm{n}=\mathbf{2 5 7}$ & \\
\hline Beta-blocker & $33,507(79.4)$ & $665(77.3)$ & $195(75.9)$ & 0.124 \\
\hline ACE inhibitor/ARB & $21,389(50.7)$ & $430(50.0)$ & $140(54.5)$ & 0.44 \\
\hline Statin & $36,918(87.5)$ & $769(89.4)$ & $210(81.7)$ & 0.005 \\
\hline Platelet inhibitor & $40,985(97.2)$ & $840(97.7)$ & $239(93.0)$ & $<0.001$ \\
\hline Dual anti-platelet inhibition & $39,318(93.2)$ & $811(94.3)$ & $228(88.7)$ & $228(88.7)$ \\
\hline Loop diuretic & 8055 (19.1) & $161(18.7)$ & $52(20.3)$ & 0.86 \\
\hline
\end{tabular}

PCl: percutaneous coronary intervention; ACE: angiotensin-converting enzyme inhibitor; ARB: angiotensin 2 receptor blocker; NSAID: Non-steroid anti inflammatory drug.

primary analysis, patients with psoriasis were more likely to receive NSAIDs, antidepressive medication, and glucose-lowering drugs. An overview of post-PCI treatment is presented in Table 2 .

\section{Post $\mathrm{PCl}$ prognosis}

Psoriasis was associated with an increased risk of both study endpoints ( $\mathrm{p}$-value for trend 0.004 and 0.007 for all cause mortality and the composite endpoint, respectively). In analyses stratified for psoriasis severity patients with severe psoriasis, but not those with mild disease had significantly increased prevalence of both study endpoints compared to patients without psoriasis. The incidence rates (IRs) and 95\% confidence intervals (CIs) for all-cause mortality were 30.5 (CI 29.7-31.3), 29.9 (CI 24.7-36.1), and 47.2 (CI 35.0-63.6) for patients without psoriasis, with mild psoriasis, and with severe psoriasis, respectively. The corresponding IRs for the composite endpoint of death, myocardial infarction, and stroke were 38.9 (CI 38.1.8-39.9), 37.3 (CI 31.4-44.4), and 55.4 (CI 41.8-73.5). In the primary analysis with start of follow-up at the time of the index PCI and adjustment for age and sex, the hazard ratios (HRs) for all cause mortality were 1.10 (CI $0.91-1.33$ ) and 1.83 (CI 1.362.47) for mild and severe psoriasis, respectively (Table 3 ). For the composite endpoint the corresponding age and sex adjusted HRs were 1.07 (CI 0.90-1.27) for mild psoriasis and 1.65 (CI 1.24-2.19) for severe psoriasis. The 
Table 3 Number of events, incidence rates, adjusted HRs, and $95 \% \mathrm{Cl}$

\begin{tabular}{|c|c|c|c|c|c|}
\hline & Number of events & Incidence rate & $95 \% \mathrm{Cl}$ & Hazard ratio & $95 \% \mathrm{Cl}$ \\
\hline \multicolumn{6}{|l|}{ All cause mortality } \\
\hline \multicolumn{6}{|c|}{ Follow-up start at index $\mathrm{PCl}$} \\
\hline Mild psoriasis & 107 & 29.9 & 24.7-36.1 & 1.10 & $0.91-1.33$ \\
\hline Severe psoriasis & 43 & 47.2 & $35.0-63.6$ & 1.67 & $1.24-2.26$ \\
\hline \multicolumn{6}{|c|}{ Follow-up start at day 30} \\
\hline Mild psoriasis & 85 & 24.3 & 19.7-30.1 & 1.13 & $0.91-1.40$ \\
\hline Severe psoriasis & 38 & 42.8 & $31.1-58.8$ & 1.78 & $1.30-2.46$ \\
\hline \multicolumn{6}{|c|}{ Composite endpoint } \\
\hline \multicolumn{6}{|c|}{ Follow-up start at index $\mathrm{PCl}$} \\
\hline Mild psoriasis & 128 & 37.3 & $31.4-44.4$ & 1.07 & $0.90-1.28$ \\
\hline Severe psoriasis & 48 & 55.4 & 41.8-73.5 & 1.54 & 1.16-2.05 \\
\hline \multicolumn{6}{|c|}{ Follow-up start at day 30} \\
\hline Mild psoriasis & 90 & 26.9 & 21.9-33.1 & 1.10 & $0.90-1.36$ \\
\hline Severe psoriasis & 38 & 45.1 & $45.132 .8-61.1$ & 1.66 & $1.21-2.28$ \\
\hline
\end{tabular}

HR: hazard ratio; Cl: confidence interval; PCl: percutaneous coronary intervention.

sensitivity analysis with start of follow-up 30 days from index PCI and inclusion of post-PCI medication in the Cox regression model gave similar results. The age- and sex-adjusted estimates for all cause mortality were HR 1.11 (CI 0.89-1.37) and HR 2.06 (CI 1.50-2.84), for mild and severe psoriasis, respectively. For the composite endpoint the corresponding age and sex adjusted HRs were 1.08 (CI 0.88-1.34) for mild psoriasis and 1.90 (CI 1.382.62) for severe psoriasis (Table 3 ).

\section{Repeat revascularization}

There was a nonsignificant trend $(\mathrm{p}=0.23)$ for increased repeat revascularization rates in patients with psoriasis compared to patients without psoriasis. IRs were 66.9 (CI 65.6-68.3), 71.3 (CI 62.2-81.6), and 75.0 (CI 57.797.5), for patients without psoriasis, with mild psoriasis, and with severe psoriasis, respectively. The corresponding adjusted HRs were 1.05 (CI 0.89-1.22) and 1.14 (CI 0.851.52) for mild and severe psoriasis, respectively.

\section{Discussion}

This nationwide study of the impact of psoriasis on prognosis following PCI demonstrated an increased risk of allcause mortality and of a composite of death, myocardial infarction, and stroke, respectively, in patients with severe disease compared to subjects without psoriasis. The postPCI prognosis in patients with mild psoriasis was comparable to that of patients without psoriasis. Furthermore, patients with severe psoriasis were less likely to receive beta-blockers, statins and platelet inhibitors after PCI.

Psoriasis and atherosclerosis are chronic inflammatory diseases characterized by T-helper (Th) 1-/Th17-driven inflammation and there is an apparent overlap of inflammatory mechanisms in these diseases [1,2]. Psoriasis has previously been associated with increased prevalence of conventional cardiovascular risk factors as well as cardiovascular morbidity and mortality [4-9,13-16]. Inflammation has also been suggested to contribute to post-PCI complications including restenosis, in-stent thrombosis and mortality [17]. Very limited data are available, however, on the impact of inflammatory diseases on the prognosis after PCI. A small study of patients with systemic lupus erythematosus demonstrated a worse 1-year outcome compared to controls while a very small study of patients with rheumatoid arthritis found no differences between groups $[18,19]$. On the other hand, the current demonstration of impaired prognosis after PCI in patients with severe psoriasis is likely to contribute to the adverse contemporary post-myocardial infarction prognosis found in patients with this disease, as well as in post-myocardial infarction subjects with systemic lupus erythematosus and rheumatoid arthritis [12,20-22].

In the present study, patients with psoriasis were more likely to receive statins, angiotensin converting enzyme (ACE) inhibitors/angiotensin 2 receptor antagonists, glucose-lowering drugs, and platelet inhibitors at baseline before index PCI than patients without psoriasis, supportive of previous reports of an increased prevalence of hypertension, hypercholesterolemia and diabetes mellitus in patients with psoriasis [7,23-29]. Also, in patients with psoriasis there was a higher proportion of NSAID users and medically managed depression compared to patients without psoriasis, both of which have been associated with impaired prognosis in patients with coronary artery disease [30-32]. After adjustments for these and other measured confounders, however, we found that severe psoriasis was a risk factor for adverse prognosis following first-time PCI, this suggests that the poor 
prognosis observed was not mainly driven by these differences in comorbidity. It is noteworthy that the proportion of high risk patients, i.e., where the index PCI was performed in the setting of MI, and that the rates of (index) primary PCI procedures (indicative of ST elevation myocardial infarction) were similar between patients with severe psoriasis and patients without psoriasis. Also, the proportion of patients treated with loop diuretics was similar irrespective of psoriasis status. This indicates a comparable distribution of severe congestive heart failure and is an important observation since we were unable to control for baseline and post-PCI left ventricular ejection fraction. Repeat revascularization rates after index PCI were also not statistically different between patients with and without psoriasis, but our study suggested differences in post-PCI pharmacological treatment, with patients with severe psoriasis receiving less beta-blockers, statins, and platelet inhibitors (Table 2). Although the reasons underlying the latter observation were not examined, significant chronic comorbidity has been associated with suboptimal post-myocardial infarction care and this particular finding may be an example of the treatment-risk paradox in coronary artery disease, i.e., that patients at increased risk receive relatively less evidence-based treatment [33]. In contrast, our previous report on prognosis after myocardial infarction in patients with psoriasis in the time period 2002-06 demonstrated no significant differences in postmyocardial infarction medical treatment, but that study included fewer patients and did not specifically evaluate the impact of psoriasis severity. Importantly, the analyses that controlled for these differences in post-PCI treatment gave results comparable to the primary analysis indicating that the impaired post-PCI prognosis in patients with severe psoriasis was not mainly driven by these differences. Taken together, the current finding of impaired post-PCI prognosis in patients with severe psoriasis therefore supports the notion that psoriasis-driven chronic inflammation per se may be an important determinant of the observed differences in post-PCI prognosis. This interpretation of the data reflects the sum of other current evidence on mechanisms underlying increased risk of cardiovascular disease in patients with chronic inflammatory diseases [3,6-11,14,15]. Accepted risk scores for prognosis after myocardial infarction may therefore underestimate the risk in these patients, as is the case for primary prevention, where traditional risk factor algorithms for coronary artery disease, e.g., the Framingham risk score, are unlikely to capture the full extent of increased risk [13,34-36].

Important strengths of the present study included the large number of participants, the ability to evaluate a psoriasis activity dose-response effect, the nationwide coverage in prospectively recorded registries, the contemporary clinical setting, and the completeness of follow-up. Furthermore, the use of nationwide registries of hospitalization data and dispensed prescriptions from all pharmacies in Denmark where healthcare is readily accessible and essentially free of charge minimized selection bias related to factors such as gender, age, socioeconomic status, healthcare insurance provision and job situation. Some limitations of our study should also be acknowledged. The results relied on the accuracy of the underlying registration in the registers, e.g., in terms of the study population and outcome measures. The register coding of revascularization procedures has not been validated but it is highly unlikely that the coding of these expensive and very specific treatments are erroneous. Also, misclassification in relation to psoriasis status is likely to be non-differential. The examined psoriasis cohort was based on prescriptions filled for topical vitamin $\mathrm{D}$ derivatives and our results may not apply to patients treated with other first-line antipsoriatic drugs, e.g., topical corticosteroids. However, there is no obvious reason to suspect important differences in baseline characteristics between patients treated with topical vitamin D or other first-line antipsoriatic drugs. Importantly, we have previously demonstrated that the criteria for the psoriasis diagnosis and severity classification in our registries were valid [14]. Because the Danish population is predominantly of Caucasian descent, the results should only be extrapolated to other ethnicities with caution. Finally, we were unable to account for some important cardiovascular risk factors, e.g., family history, obesity, and smoking, and other prognostic variables including coronary pathologic findings, PCI procedural variables, and left ventricular ejection fraction, and we cannot exclude the possibility unmeasured and/or residual confounding contributed to our findings. However, information on strong predictors of post-PCI adverse events, i.e., hypertension, diabetes mellitus, renal failure, and adherence to dual anti-platelet therapy were included [37].

\section{Conclusion}

This first study of the post-PCI prognosis in patients with psoriasis demonstrated an increased risk of mortality and adverse cardiovascular events in patients with severe psoriasis compared to patients without psoriasis. These findings emphasize the increased burden of cardiovascular disease in patients with psoriasis and they call for further studies in this area of research.

\section{Methods}

The study was a retrospective longitudinal cohort study using Danish nationwide prospectively recorded registries. The study complied with the Strengthening the Reporting of Observational Studies in Epidemiology (STROBE) recommendations [38]. 


\section{Ethics}

This study was approved by The Danish Data Protection Agency (ref. 2008-41-2685). Approval from an ethics committee is not required for administrative register studies in Denmark.

\section{Study population and data sources}

The present study population comprised all subjects from the entire Danish population, who underwent a first-time PCI (Nordic classification of surgical procedures code KFNG) in the period 2002-2009. Primary PCI in patients with ST elevation myocardial infarction was identified by the procedural codes KFNG02A and KFNG05A. Patients were followed until December 31 2009 , emigration, or to the occurrence of an endpoint. Patients with PCI prior to the start of the study period were excluded. All Danish citizens have a unique lifelong personal civil registration number that enables linkage of information on an individual level across nationwide prospectively recorded registries. All medications dispensed from pharmacies were obtained from the Danish Registry of Medicinal Product Statistics, in which all dispensed prescriptions since 1995 are registered. Morbidity was obtained from the Danish National Patient Register in which hospital admissions, procedures, and diagnoses have been recorded since 1978 with the International Classification of Diseases (ICD) codes. Deaths were identified from the Central Population Register, wherein all deaths are recorded within 2 weeks. Socioeconomic status was defined as average yearly income in a period of up to 5 years prior to inclusion and divided into quintiles.

\section{Psoriasis identification}

Patients with mild psoriasis were identified according to prescriptions claimed prior to study inclusion (index PCI) for topical treatment used exclusively for psoriasis, i.e., topical vitamin-D derivatives (ATC D05AX). Subjects with severe psoriasis were identified by hospitalizations for psoriasis and psoriatic arthritis (ICD-10 L40 and M07.0-M07.3), respectively, from 1994 to index PCI, as validated previously $[9,14]$.

\section{Medical treatment and comorbidity}

Medical treatment for cardiovascular diseases and cardiovascular risk factors, including hypertension, dyslipidemia, and diabetes mellitus were identified by prescriptions for platelet inhibitors (ATC code: B01AC), beta-blockers (C07), ACE inhibitors/angiotensin 2 receptor antagonists (C09), loop diuretics (C03C), spironolactone (C03D), statins (C10A), and glucose-lowering drugs (A10) claimed up to 6 months prior to index PCI. Use of NSAID (M01A) and anti-depressive medication (N06A) was also ascertained. Initiation and adherence to cardiovascular secondary prophylaxis was evaluated at 30,180, and 360 days from the index PCI. Comorbidity was defined by hospitalizations up to 12 months prior to the index PCI according to the Ontario myocardial infarction mortality prediction rule as adapted to ICD 10 [39].

\section{Outcomes}

The primary study endpoint was all-cause mortality and a secondary composite endpoint was death, recurrent myocardial infarction (ICD-10 I21-I22) and stroke (ICD10 I60, I61, I63, and I64). Repeat revascularization rates after index PCI were also determined, including PCI and coronary artery bypass grafting. The myocardial infarction and stroke diagnoses in the Danish registries have previously been found valid [40,41].

\section{Statistical analysis}

Baseline characteristics were summarized and presented as means with standard deviations (SD) and percentages. Differences in baseline characteristics were assessed by analyses of variance (ANOVA) and by chi-square test for categorical covariates. IRs of study endpoints were reported as events per 1000 patient-years. The HRs and corresponding CIs were estimated by Cox regression models controlling for confounding factors including calendar year, patient age, gender, baseline use of medication and comorbidity, and socioeconomic data. A sensitivity analysis with start of follow-up 30 days from the index PCI and inclusion of post-PCI medication in the regression model was also done in order to assess the impact of differences in post-PCI treatment between the groups. Age- and sex-adjusted models, and a fully adjusted model with inclusion of age, sex, medication, comorbidity, and socioeconomic status, were fitted. Model assumptions, including absence of interaction between model covariates and the proportional hazard assumption were tested and found to be valid. A two-sided $\mathrm{p}<0.05$ was considered statistical significant. All statistical analyses were performed with the SAS statistical software version 9.2 (Sas Institute inc., Cary, NC, USA) and Stata software version 11 (Statacorp, College st., TX, USA).

\section{Abbreviations}

PCl: Percutaneous coronary intervention; IR: Incidence rate; HR: Hazard ratio; Cl: 95\% Confidence interval; NSAID: Non steroid anti-inflammatory drugs; ACE: Angiotensin-converting enzyme inhibitor; ARB: Angiotensin 2 receptor blocker.

Competing interests

The authors have no competing interests.

Authors' contributions

$\mathrm{OA}, J \mathrm{~L}, \mathrm{GG}, \mathrm{LS}, \mathrm{CTP}$, and PRH perceived and designed the study. OA, JL, and $\mathrm{PRH}$ drafted the manuscript. OA, JL, GG, and CTP carried out the statistical 
analyses. OA, JL, GG, JBO, MC, LS, CTP, and PRH participated in interpretation of the data, critical revision of the manuscript, and decision to submit for publication. All authors read and approved the final manuscript.

\section{Author details}

'Department of Cardiology, Copenhagen University Hospital, Gentofte, Hellerup DK-2900, Denmark. ${ }^{2}$ Department of Cardiology, Copenhagen University Hospital, Roskilde, Roskilde DK-4000, Denmark. ${ }^{3}$ Department of Dermatology, Copenhagen University Hospital, Gentofte, Hellerup DK-2900, Denmark.

Received: 8 February 2012 Accepted: 20 September 2012

Published: 24 September 2012

\section{References}

1. Nestle FO, Kaplan DH, Barker J: Psoriasis. N Engl J Med 2009, 361:496-509.

2. Libby P, Ridker PM, Hansson GK: Inflammation in atherosclerosis: from pathophysiology to practice. J Am Coll Cardiol 2009, 54:2129-2138.

3. Frostegard J: Atherosclerosis in patients with autoimmune disorders. Arterioscler Thromb Vasc Biol 2005, 25:1776-1785.

4. Friedewald VE, Cather JC, Gelfand JM, Gordon KB, Gibbons GH, et al: AJC editor's consensus: psoriasis and coronary artery disease. Am J Cardiol 2008, 102:1631-1643

5. Wakkee M, Thio HB, Prens EP, Sijbrands EJ, Neumann HA: Unfavorable cardiovascular risk profiles in untreated and treated psoriasis patients. Atherosclerosis 2007, 190:1-9.

6. Gelfand JM, Dommasch ED, Shin DB, Azfar RS, Kurd SK, et al The risk of stroke in patients with psoriasis. J Invest Dermatol 2009, 129:2411-2418.

7. Gelfand JM, Neimann AL, Shin DB, Wang X, Margolis DJ, et al: Risk of myocardial infarction in patients with psoriasis. JAMA 2006, 296:1735-1741.

8. Mehta NN, Azfar RS, Shin DB, Neimann AL, Troxel AB, et al: Patients with severe psoriasis are at increased risk of cardiovascular mortality: cohort study using the General Practice Research Database. Eur Heart J 2010, 31:1000-1006

9. Ahlehoff $\mathrm{O}$, Gislason $\mathrm{GH}$, Charlot M, Jorgensen $\mathrm{CH}$, Lindhardsen J, et al: Psoriasis is associated with clinically significant cardiovascular risk: a Danish nationwide cohort study. J Intern Med 2011, 270:147-157.

10. Lindhardsen J, Ahlehoff O, Gislason GH, Madsen OR, Olesen JB, et al: The risk of myocardial infarction in rheumatoid arthritis and diabetes mellitus: a Danish nationwide cohort study. Ann Rheum Dis 2011, 70:929-934.

11. Meesters EW, Hansen H, Spronk HM, Hamulyak K, Rosing J, et al: The inflammation and coagulation cross-talk in patients with systemic lupus erythematosus. Blood Coagul Fibrinolysis 2007, 18:21-28.

12. Ahlehoff $\mathrm{O}$, Gislason $\mathrm{GH}$, Lindhardsen J, Olesen JB, Charlot M, et al: Prognosis following first-time myocardial infarction in patients with psoriasis: a Danish nationwide cohort study. J Intern Med 2011, 270:237-244.

13. Gisondi P, Farina S, Giordano MV, Girolomoni G: (2010) Usefulness of the Framingham Risk Score in Patients With Chronic Psoriasis. Am J Cardiol 2010, 106:1754-1757.

14. Ahlehoff $\mathrm{O}$, Gislason $\mathrm{GH}$, Jorgensen $\mathrm{CH}$, Lindhardsen J, Charlot M, et al: (2011) Psoriasis and risk of atrial fibrillation and ischaemic stroke: a Danish Nationwide Cohort Study. Eur Heart J 2012, 33:2054-2064.

15. Ahlehoff O, Gislason GH, Lindhardsen J, Charlot MG, Jorgensen $\mathrm{CH}$, et al: Psoriasis carries an increased risk of venous thromboembolism: a Danish nationwide cohort study. PLoS One 2011, 6:e18125.

16. Gulliver WP, Macdonald D, Gladney N, Alaghehbandan R, Rahman P, et al: Long-term prognosis and comorbidities associated with psoriasis in the Newfoundland and Labrador founder population. J Cutan Med Surg 2011, 15:37-47.

17. Park DW, Yun SC, Lee JY, Kim WJ, Kang SJ, et al: C-reactive protein and the risk of stent thrombosis and cardiovascular events after drug-eluting stent implantation. Circulation 2009, 120:1987-1995.

18. Maksimowicz-McKinnon K, Selzer F, Manzi S, Kip KE, Mulukutla SR, et al: Poor 1 -year outcomes after percutaneous coronary interventions in systemic lupus erythematosus: report from the National Heart, Lung, and Blood Institute Dynamic Registry. Circ Cardiovasc Interv 2008, 1:201-208.

19. Desai SP, Januzzi JL, Pande AN, Pomerantsev EV, Resnic FS, et al: Comparison of symptoms, treatment, and outcomes of coronary artery disease among rheumatoid arthritis and matched subjects undergoing percutaneous coronary intervention. Semin Arthritis Rheum 2010, 40:215-221.

20. Shah MA, Shah AM, Krishnan E: Poor outcomes after acute myocardial infarction in systemic lupus erythematosus. J Rheumatol 2009, 36:570-575.

21. Douglas KM, Pace AV, Treharne GJ, Saratzis A, Nightingale P, et al: Excess recurrent cardiac events in rheumatoid arthritis patients with acute coronary syndrome. Ann Rheum Dis 2006, 65:348-353.

22. Sodergren A, Stegmayr B, Lundberg V, Ohman ML, Wallberg-Jonsson S: Increased incidence of and impaired prognosis after acute myocardia infarction among patients with seropositive rheumatoid arthritis. Ann Rheum Dis 2007, 66:263-266.

23. Boehncke S, Thaci D, Beschmann H, Ludwig RJ, Ackermann H, et al: Psoriasis patients show signs of insulin resistance. Br J Dermatol 2007, 157:1249-1251.

24. Cohen AD, Gilutz H, Henkin Y, Zahger D, Shapiro J, et al: Psoriasis and the metabolic syndrome. Acta Derm Venereol 2007, 87:506-509.

25. Azfar RS, Gelfand JM: Psoriasis and metabolic disease: epidemiology and pathophysiology. Curr Opin Rheumatol 2008, 20:416-422.

26. Cohen AD, Dreiher J, Shapiro Y, Vidavsky L, Vardy DA, et al: Psoriasis and diabetes: a population-based cross-sectional study. J Eur Acad Dermatol Venereol 2008, 22:585-589.

27. Kaye JA, Li L, Jick SS: Incidence of risk factors for myocardial infarction and other vascular diseases in patients with psoriasis. Br J Dermatol 2008, 159:895-902.

28. Kimball AB, Robinson D Jr, Wu Y, Guzzo C, Yeilding N, et al: Cardiovascular disease and risk factors among psoriasis patients in two US healthcare databases, 2001-2002. Dermatology 2008, 217:27-37.

29. Gladman DD, Ang M, Su L, Tom BD, Schentag CT, et al: Cardiovascular morbidity in psoriatic arthritis. Ann Rheum Dis 2009, 68:1131-1135.

30. Gislason GH, Jacobsen S, Rasmussen JN, Rasmussen S, Buch P, et al: Risk of death or reinfarction associated with the use of selective cyclooxygenase- 2 inhibitors and nonselective nonsteroidal antiinflammatory drugs after acute myocardial infarction. Circulation 2006, 113:2906-2913.

31. Ray WA, Varas-Lorenzo C, Chung CP, Castellsague J, Murray KT, et al: Cardiovascular risks of nonsteroidal antiinflammatory drugs in patients after hospitalization for serious coronary heart disease. Circ Cardiovasc Qual Outcomes 2009, 2:155-163.

32. Celano CM, Huffman JC: Depression and cardiac disease: a review. Cardiol Rev 2011, 19:130-142.

33. McAlister FA, Oreopoulos A, Norris CM, Graham MM, Tsuyuki RT, et al: Exploring the treatment-risk paradox in coronary disease. Arch Intern Med 2007, 167:1019-1025.

34. Chung CP, Oeser A, Avalos I, Raggi P, Stein CM: Cardiovascular risk scores and the presence of subclinical coronary artery atherosclerosis in women with systemic lupus erythematosus. Lupus 2006, 15:562-569.

35. Kimhi O, Caspi D, Bornstein NM, Maharshak N, Gur A, et al: Prevalence and risk factors of atherosclerosis in patients with psoriatic arthritis. Semin Arthritis Rheum 2007, 36:203-209.

36. Tam LS, Shang Q, Li EK, Tomlinson B, Chu TT, et al: Subclinical carotid atherosclerosis in patients with psoriatic arthritis. Arthritis Rheum 2008 59:1322-1331.

37. Jukema JW, Verschuren JJ, Ahmed TA, Quax PH: Restenosis after PCI. Part 1: pathophysiology and risk factors. Nat Rev Cardiol 2012 9:53-62.

38. von Elm E, Altman DG, Egger M, Pocock SJ, Gotzsche PC, et al: The Strengthening the Reporting of Observational Studies in Epidemiology (STROBE) statement: guidelines for reporting observational studies. Lancet 2007, 370:1453-1457.

39. Vermeulen MJ, Tu JV, Schull MJ: ICD-10 adaptations of the Ontario acute myocardial infarction mortality prediction rules performed as well as the original versions. J Clin Epidemiol 2007, 60:971-974. 
40. Madsen M, Davidsen M, Rasmussen S, Abildstrom SZ, Osler M: The validity of the diagnosis of acute myocardial infarction in routine statistics: a comparison of mortality and hospital discharge data with the Danish MONICA registry. J Clin Epidemiol 2003, 56:124-130.

41. Krarup LH, Boysen $G$, Janjua $H$, Prescott $E$, Truelsen T: Validity of stroke diagnoses in a National Register of Patients. Neuroepidemiology 2007, 28:150-154.

doi:10.1186/1471-2261-12-79

Cite this article as: Ahlehoff et al:: Prognosis after percutaneous

coronary intervention in patients with psoriasis: a cohort study using

Danish nationwide registries. BMC Cardiovascular Disorders 2012 12:79.

\section{Submit your next manuscript to BioMed Central and take full advantage of:}

- Convenient online submission

- Thorough peer review

- No space constraints or color figure charges

- Immediate publication on acceptance

- Inclusion in PubMed, CAS, Scopus and Google Scholar

- Research which is freely available for redistribution 